\title{
A Case of Hereditary Hemorrhagic Relangiectasia Suspected due to Anemia during Anticoagulation Therapy after Thrombectomy
}

Naoyuki Arai, ${ }^{1}$ Hiroshi Kizuki, ${ }^{2}$ Kei Yamazaki, ${ }^{1}$ Mami Akiyama, ${ }^{2}$ Hiroaki Arai, $^{2}$ and Hidetoshi Kasuya ${ }^{1}$

Objective: Hereditary hemorrhagic telangiectasia $(\mathrm{HHT})$ may be associated with paradoxical cerebral embolism caused by pulmonary arteriovenous malformation (PAVM). We present a case of $\mathrm{HHT}$ diagnosed by progressive anemia during anticoagulant therapy following mechanical thrombectomy.

Case Presentation: The patient was a 59-year-old woman who presented with acute stroke due to intracranial large vessel occlusion. Mechanical thrombectomy was successfully performed and the thrombus was retrieved. Postoperatively, anticoagulant therapy was started; however, she developed progressive anemia, which was associated with marked weakness, although no bleeding source was detected. Thorough postoperative imaging studies revealed PAVMs, which may be a source of cerebral embolism. It was noted that she frequently had episodes of epistaxis and a family history of PAVM. Embolization of PAVMs was performed to prevent the recurrence of embolic disorders. After this procedure, anticoagulant therapy was safely discontinued, which resulted in the improvement of anemia.

Conclusion: Physicians need to consider the possibility of HHT associated with PAVM which can cause paradoxical cerebral embolism.

Keywords hereditary hemorrhagic telangiectasia, pulmonary arteriovenous malformation, paradoxical cerebral embolism, endovascular thrombectomy

\section{Introduction}

It is difficult to evaluate diseases that cause acute occlusion of major cerebral arteries immediately after onset in the absence of atrial fibrillation. However, when thrombectomy is successful or when there is no residual stenosis of the artery after thrombectomy, anticoagulant therapy is selected early after surgery in many cases, considering the possibility of cardiogenic cerebral embolism related to atrial fibrillation, ${ }^{1)}$

$\overline{{ }^{I} \text { Department of Neurosurgery, Tokyo Women's Medical University }}$ Medical Center East, Tokyo, Japan

${ }^{2}$ Department of Neurosurgery, Toda Chuo General Hospital, Saitama, Japan

Received: May 14, 2019; Accepted: October 25, 2019 Corresponding author: Arai Naoyuki. Department of Neurosurgery, Tokyo Women's Medical University Medical Center East, 2-1-10 Nishiogu, Arakawa-ku, Tokyo 116-8567, Japan

Email: naoyukisensei0816@gmail.com

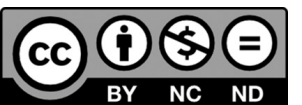

This work is licensed under a Creative Commons Attribution-NonCommercialNoDerivatives International License.

(C)2020 The Japanese Society for Neuroendovascular Therapy which accounts for approximately $70 \%$ of causative diseases, or paradoxical cerebral embolism. However, universal examinations for embolic stroke of undetermined source (ESUS) may not lead to a diagnosis of a specific disease, ${ }^{2-4}$ ) and it is difficult to detect latent atrial fibrillation. ${ }^{5-7)}$ Therefore, if the prevention of recurrence by continuous anticoagulant therapy is overestimated, adhering to latent atrial fibrillation, an originally causative disease may be overlooked. Some diseases that cause cerebral embolism involve hemorrhagic diathesis, represented by hereditary hemorrhagic telangiectasia (HHT). The continuation of anticoagulant therapy may increase the risk of hemorrhage. In this study, we report a patient in whom anticoagulant therapy after thrombectomy induced anemia, leading to a diagnosis of HHT, and review the literature.

\section{Case}

Patient: A 59-year-old female.

Medical history: At the age of 42 years, a diagnosis of iron-deficiency anemia was made and an iron preparation was orally administered. 

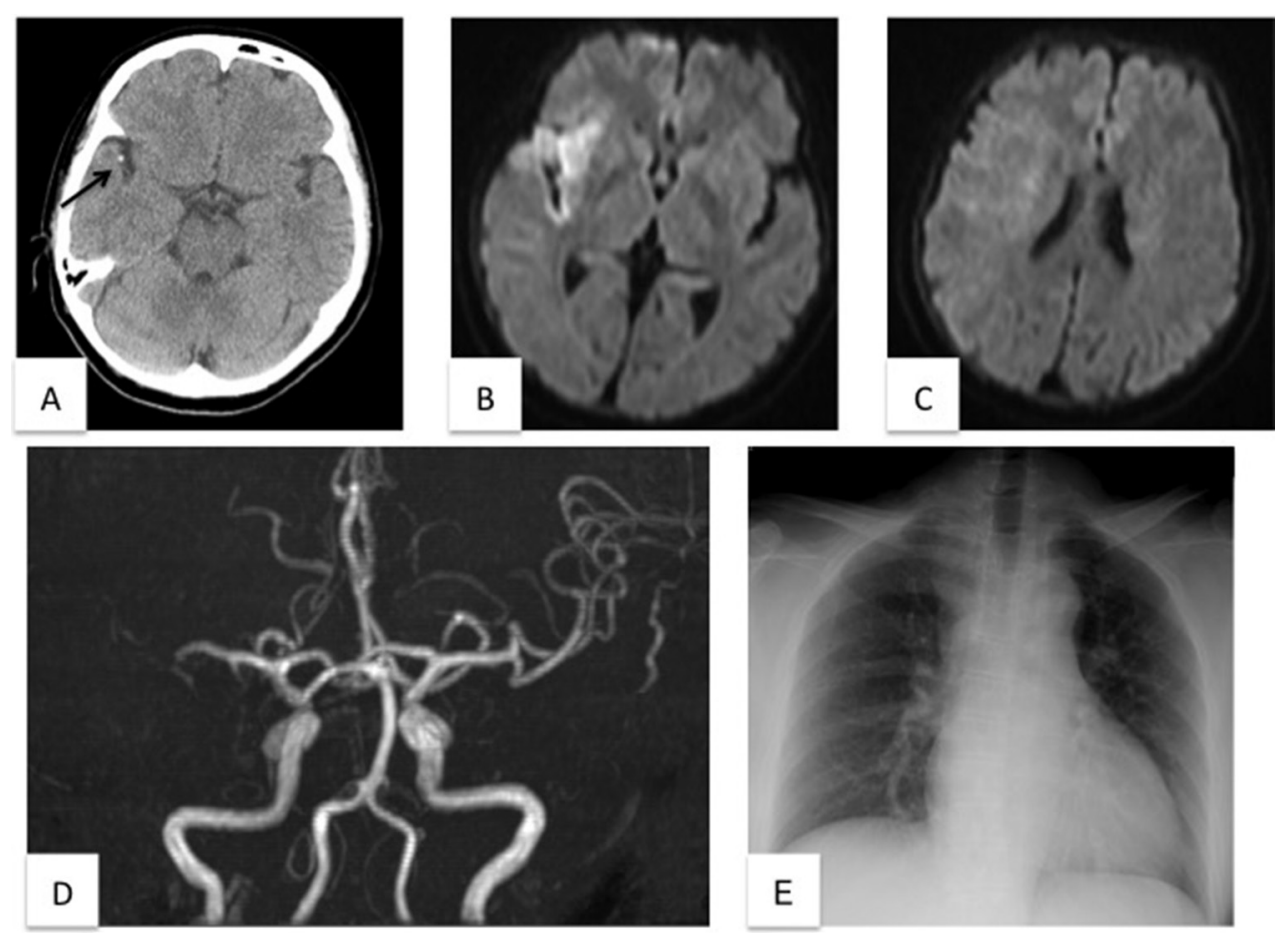

Fig. 1 Images on arrival. (A) Head CT revealed a hyper-dense sign in the right MCA region (black arrow). (B and C) Diffusion-weighted cephalic MRI showed high signal intensity in the right insular cortex and temporal lobe. (D) Cephalic MRA revealed occlusion of the right MCA. (E) Chest X-ray film on admission. At this point, the presence of a pulmonary arteriovenous fistula was not suspected. MCA: middle cerebral artery; MRA: magnetic resonance angiography

Lifestyle/family history: Not contributory.

Present illness: After waking-up, weakness of the left half body suddenly developed while washing the face. She was brought to our hospital by ambulance.

Physical examination on the initial consultation: The Japan Coma Scale score was I-1. Her pulse was 80 beats $/ \mathrm{min}$, being regular. The $\mathrm{SPO}_{2}$ was $97 \%$ (room air). Right conjugate deviation, left facial droop, dysarthria, and left hemiparesis were observed. The National Institutes of Health Stroke Scale (NIHSS) score was 8.

Laboratory data on admission: Hemoglobin (Hb), hematocrit $(\mathrm{Ht})$, and mean corpuscular volume (MCV) were $8.0 \mathrm{~g} / \mathrm{dL}$, $28.2 \%$, and $90.7 \%$, respectively, suggesting normocytic normochromic anemia. The D-dimer level was 0.37 , being normal. The arterial blood gas test did not reveal any marked abnormalities (room air: $\mathrm{PaO}_{2}: 87.7 \mathrm{mmHg}, \mathrm{SPO}_{2}, 96.1 \%$ ). Imaging findings: Cephalic computed tomography (CT) demonstrated a hyper-dense middle cerebral artery (MCA) sign in the right MCA region (Fig. 1A). High signal intensity was observed in the right insular cortex/temporal lobe on cephalic magnetic resonance imaging (MRI)-diffusionweighted imaging (DWI) (Fig. 1B and 1C). The DWIAlberta Stroke Program Early CT Score (ASPECTS) was
8/10. Cephalic magnetic resonance angiography (MRA) revealed occlusion of the M1 right MCA (Fig. 1D). Furthermore, chest X-ray did not demonstrate any abnormalities (Fig. 1E). Intravenous thrombolysis was started 150 minutes after onset. Subsequently, cerebral angiography was performed. Occlusion of the distal M1 right MCA persisted (Fig. 2A), and thrombectomy was conducted. Endovascular treatment: Under local anesthesia, a 9Fr long sheath was inserted into the right femoral artery. A 9Fr Optimo (Tokai Medical Products, Aichi, Japan) was inserted to the A 9Fr Optimo (Tokai Medical Products) was navigated to the right internal carotid artery. A Penumbra ACE 68 (Penumbra Inc, Alameda, CA, USA) was inserted to the proximal occluded site of the right MCA coaxially to a CHIKAI 14 (Asahi Intecc Co., Ltd., Aichi, Japan) and Marksman micro catheter (Medtronic, Minneapolis, MN, USA). Using the CHIKAI 14, the Marksman micro catheter was guided into the M2 superior trunk of the right MCA. A Trevo XP (Stryker, Kalamazoo, MI, USA) measuring $4 \times 20 \mathrm{~mm}$ was deployed at a occluded vessel, and a red thrombus measuring approximately $10 \mathrm{~mm}$ was continuously aspirated/collected through the Penumbra ACE 68 (Fig. 2B). Recanalization (Thrombolysis in Cerebral 


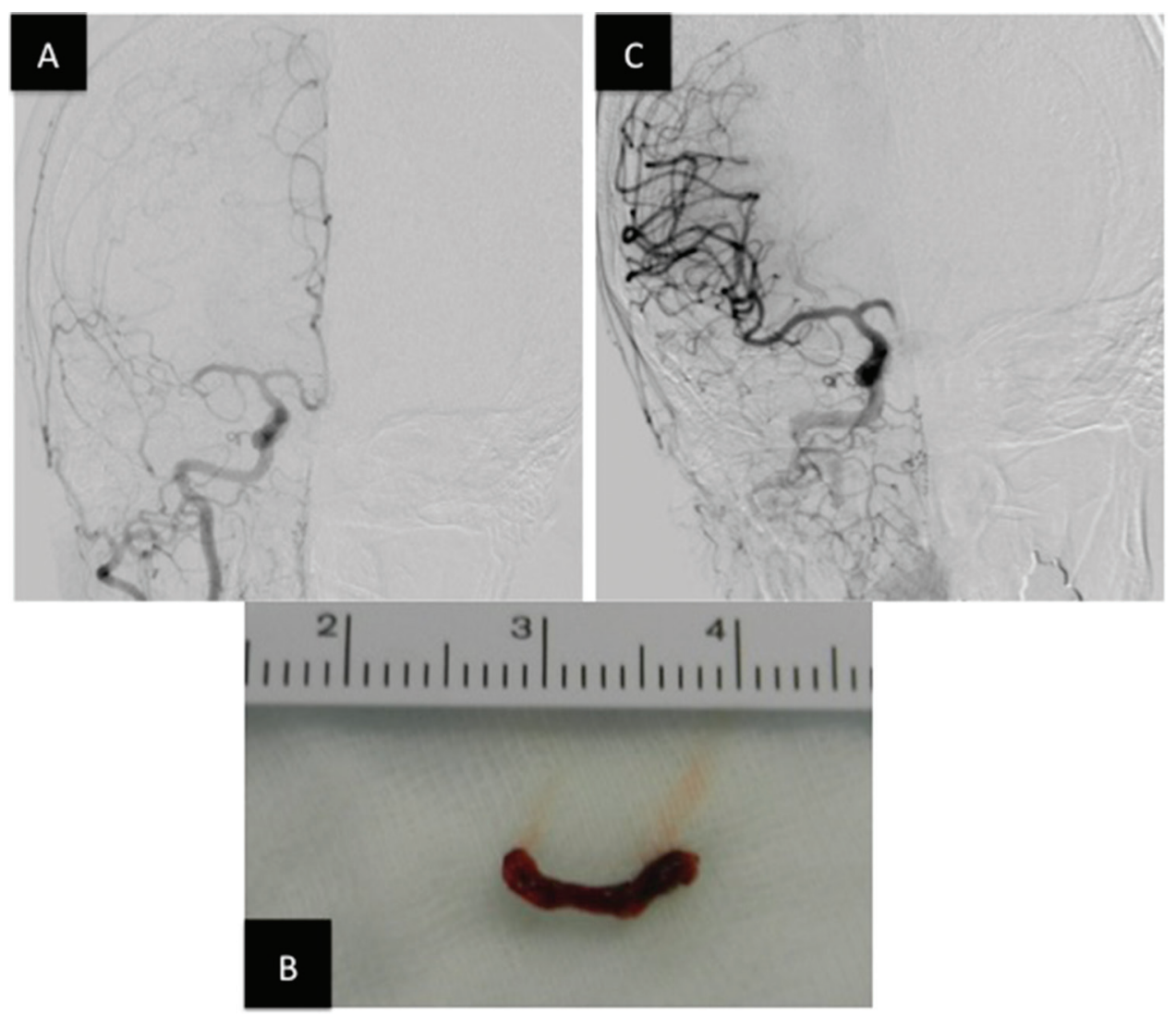

Fig. 2 Images before and after thrombectomy. (A) Right common carotid angiography before thrombectomy. Occlusion of the M1 right MCA was observed. (B) A photograph of a red thrombus, measuring approximately $10 \mathrm{~mm}$, extirpated during thrombectomy. (C) Right internal carotid angiography after thrombectomy. Recanalization (Thrombolysis in Cerebral Infarction grade: 3 ) was achieved. MCA: middle cerebral artery

Infarction grade 3) was achieved 270 minutes after onset (Fig. 2C).

Postoperative course: The symptoms improved immediately after surgery. Neither cephalic MRI nor MRA suggested marked deterioration of infarction or hemorrhagic changes 2 days after surgery (Fig. 3A-3C). Occluded vessel recanalization was maintained (Fig. 3D), and the NIHSS score was 1 . $\mathrm{Hb}$ and $\mathrm{Ht}$ were $7.6 \mathrm{~g} / \mathrm{dL}$, and $26.8 \%$, respectively; there was no marked decrease. The administration of an iron preparation, which had been orally administered before admission, was resumed. On the same day, heparin administration was started. Simultaneously, detailed examination for the source of embolism was conducted. There was no thrombus on transthoracic echocardiography or ultrasonography of the lower limb veins. Atrial fibrillation was not detected on Holter's electrocardiography or extracorporeal electrocardiographic monitoring. Furthermore, there was no occlusive major artery disease, congenital coagulation disorders, angitis syndrome, or autoimmune disease such as anti-phospholipid syndrome. Severe weakness suddenly developed 7 days after surgery. Considering recurrent embolism, cephalic MRI/MRA was performed, but there was no new infarction or occlusion of a cerebral artery (Fig. 4A-4C). However, the $\mathrm{Hb}$ and $\mathrm{Ht}$ values were $6.2 \mathrm{~g} / \mathrm{dL}$ and $19.1 \%$, respectively, suggesting the progression of anemia. There was no apparent major bleeding at any site, including the site of puncture. The activated partial thromboplastin time was 42.9 seconds; there was no excessive prolongation in comparison with the value before the start of heparin administration (26.8 seconds). To examine the source of hemorrhage, contrast-enhanced CT of the thorax to pelvis was performed. There was no site of hemorrhage, but pulmonary arteriovenous fistulae were detected in the bilateral lung fields, suggestive of the source of embolism (cerebral infarction) (Fig. 5A-5C). We considered the severe weakness to be related to a reduction in the circulating blood volume. After the administration of 4 units of blood transfusion, the symptoms promptly improved. When explaining the results of examination, we heard that the patient's son had undergone treatment for a 

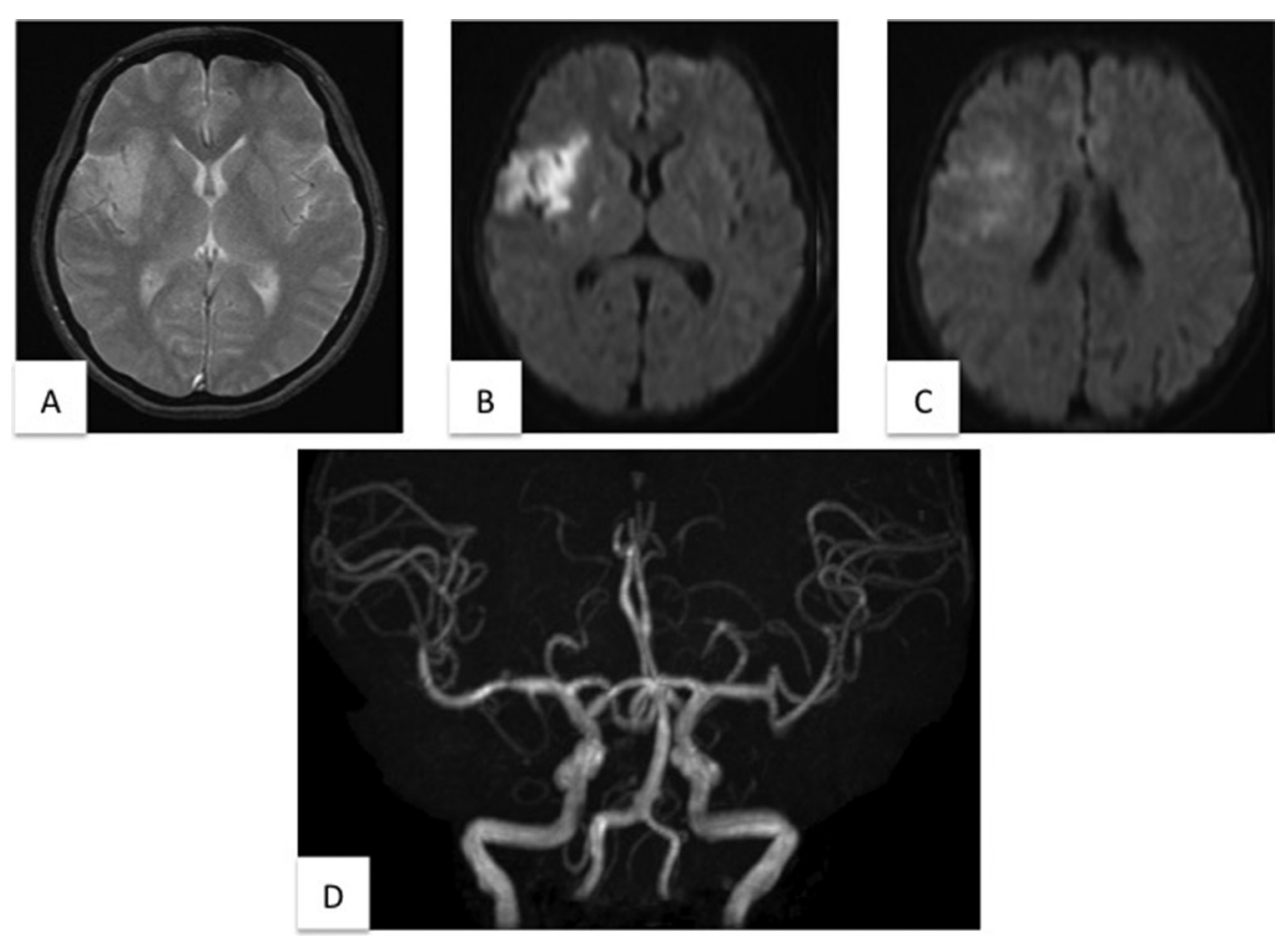

Fig. 3 Cephalic MRI/MRA findings on the 2nd postoperative day. (A) T2*-weighted cephalic MRI did not show hemorrhagic change. (B and C) Diffusion-weighted cephalic MRI demonstrated minor cerebral infarction in the right internal capsule in comparison with the preoperative images. (D) Cephalic MRA confirmed that the patency of the right MCA was maintained. MCA: middle cerebral artery; MRA: magnetic resonance angiography

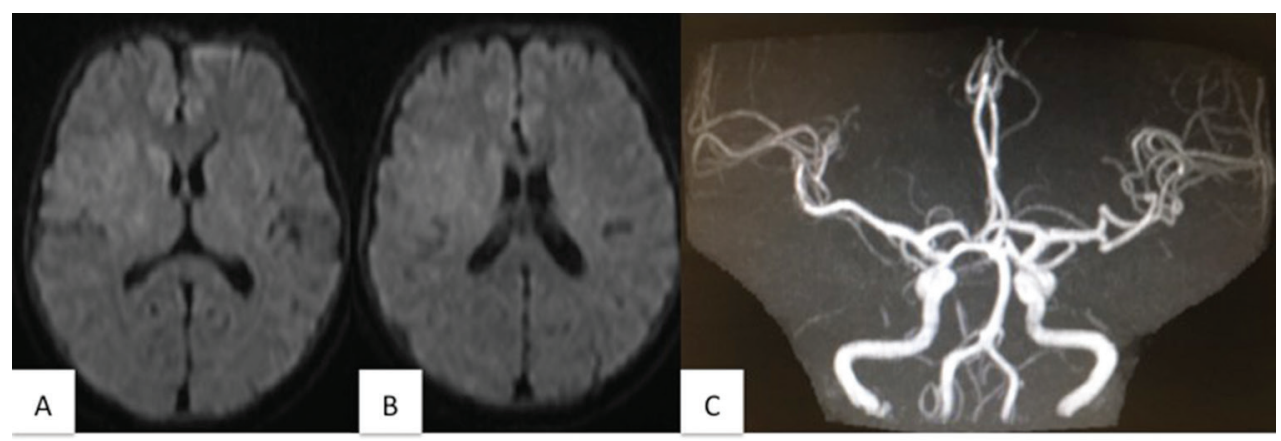

Fig. 4 Cephalic MRI/MRA findings on a change in the condition on the 7th postoperative day. (A and B) Diffusion-weighted cephalic MRI did not show any high-signal-intensity area. (C) Cephalic MRA did not reveal any new occlusion. MRA: magnetic resonance angiography

pulmonary arteriovenous fistula, and that he had a history of repeated nasal hemorrhage. Furthermore, the patient also had a history of repeated nasal hemorrhage. Cerebral embolism and anemia as an underlying disease suggested the possibility of HHT. As a fecal blood test was positive, nasal cavity assessment and gastrointestinal endoscopy were conducted for detailed examination of anemia and HHT diagnosis. There was no active hemorrhage, but telangiectasia of the nasal mucosa, antrum of the stomach, and ascending colon was noted (Fig. 5D and 5E). The patient met three of Curacao's diagnostic criteria: repeated nasal hemorrhage, nasal telangiectasia, and pulmonary arteriovenous malformation, leading to a definitive diagnosis of HHT. Furthermore, the progression of anemia was considered to be associated with the anticoagulant-therapy-related deterioration of gastrointestinal hemorrhage. To prevent recurrent cerebral infarction and complete anticoagulant therapy, catheter embolization of the pulmonary arteriovenous fistulae was performed at another hospital (Fig. 6A to 6F). Fine pulmonary arteriovenous fistulae, which were unable 


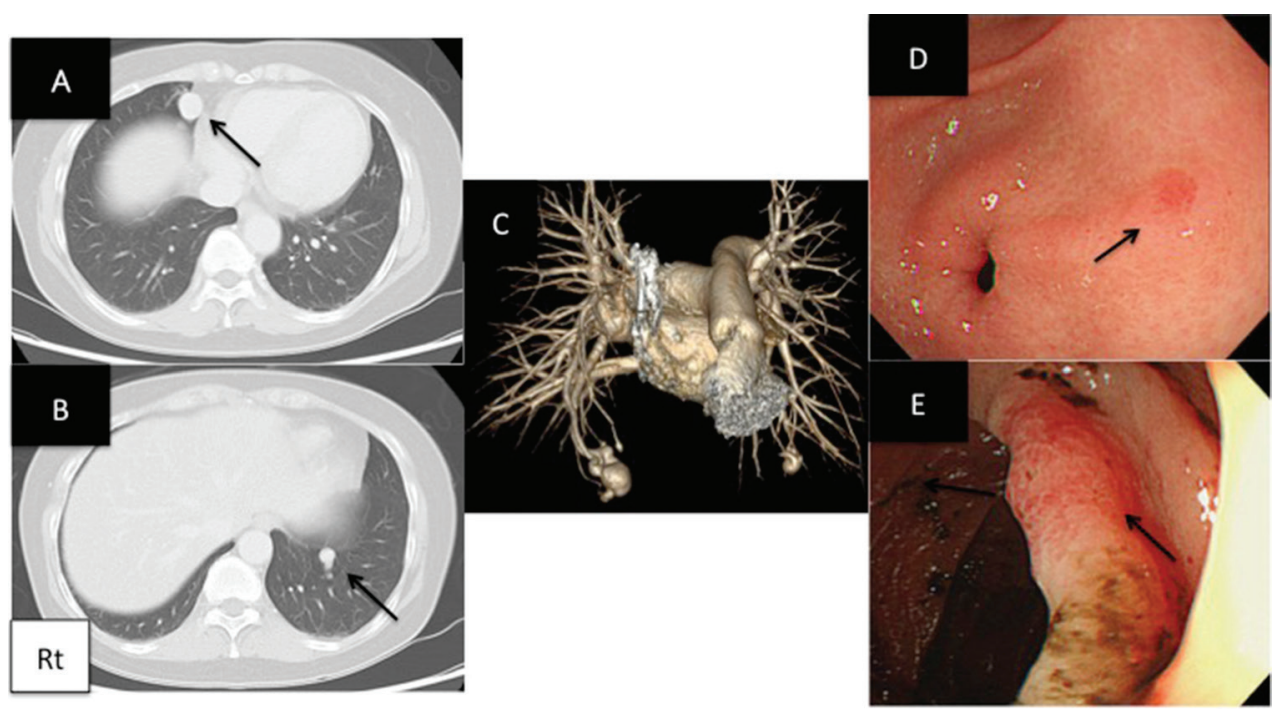

Fig. 5 Images of pulmonary arteriovenous fistulae and upper/lower gastrointestinal endoscopy. (A) An axial section on thoracic CT showed a pulmonary arteriovenous fistula in the $\mathrm{S} 5$ area of the right middle lung lobe (black arrow). (B) An axial section of thoracic CT showed a pulmonary arteriovenous fistula in the S9 area of the left inferior lung lobe (black arrow). (C) Thoracic 3D-CT angiography revealed pulmonary arteriovenous fistulae with feeding artery diameters of 4.7 and $3.1 \mathrm{~mm}$ in the right middle and left inferior lung lobes, respectively. (D) Upper gastrointestinal endoscopy showed capillary telangiectasia of the anterior gastric wall (black arrow). (E) Lower gastrointestinal endoscopy showed capillary telangiectasia of the ascending colon (black arrow). 3D-CT: three-dimensional computed tomography
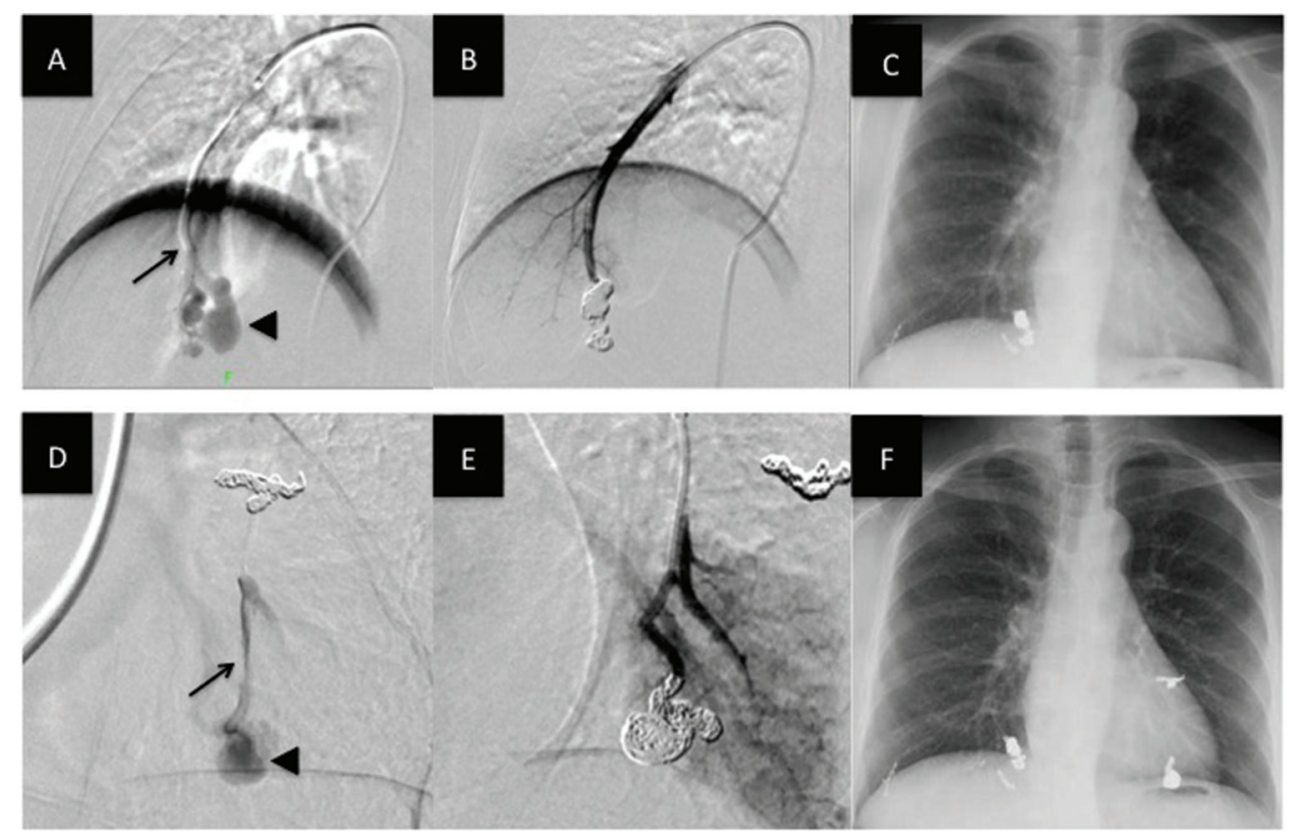

Fig. 6 Selective pulmonary arteriography for pulmonary arteriovenous fistulae and post-treatment images. (A) Selective pulmonary arteriography of the right lung. Selective imaging of an S9 feeding artery was conducted (black arrow). Varix formation was noted (black arrow head). (B) After embolization, the disappearance of the shunt was observed. (C) Postoperative chest X-ray film. In the S8 and S10 areas, fine pulmonary arteriovenous fistulae were also observed and embolization was performed. (D) Selective pulmonary arteriography of the left lung. Selective imaging of an S9 feeding artery was performed (black arrow). Varix formation was noted (black arrow head). (E) After embolization, the disappearance of the shunt was observed. (F) Postoperative chest X-ray film. In the S6 area, a fine pulmonary arteriovenous fistula was also observed, and embolization was performed. 
to be confirmed on three-dimensional (3D)-computed tomography angiography (CTA), were detected at the time of treatment, and catheter embolization of all fistulae was performed. There was no residual pulmonary arteriovenous fistula, leading to the completion of anticoagulant therapy. During the 1-year follow-up, there has been no recurrent pulmonary arteriovenous fistula or cerebral embolism. There has been no progression of anemia.

\section{Discussion}

Yoshimura et al. previously reported that acute-phase major cerebral artery occlusion is related to atherothrombotic cerebral infarction in approximately $20 \%$ of patients, and that other etiological factors or those that cannot be classified were involved in $9 \%$. Such occlusion is associated with etiological factors other than cardiogenic embolism in approximately $30 \%$. On the other hand, the risk of cardiogenic cerebral embolism is approximately $70 \% 1$ ); therefore, if mechanical thrombectomy is successful or if there is no residual stenosis of the artery after thrombectomy, cardiogenic cerebral embolism must be considered.

When the etiology of symptomatic cerebral infarction is unclear, it is necessary to evaluate the condition using the diagnostic criteria for ESUS: (1) identification of non-lacunar infarction using CT or MRI, (2) absence of occlusion or $\geq 50 \%$ stenosis of infracted-focus-associated cervical or cerebral arteries, (3) absence of high-risk heart disease that may cause embolism, and (4) absence of specific etiological factors that may cause cerebral infarction (angitis, arterial dissociation, migraine, vasospasm, and incorrect drug use). ${ }^{2,4)}$ Our patient met the above criteria, leading to a diagnosis of ESUS. A consensus regarding the selection of antiplatelet or anticoagulant therapies for ESUS has not been reached. ${ }^{4)}$ However, one study suggested that ESUS was caused by covert atrial fibrillation in approximately $40 \%$ of patients. ${ }^{7)}$ Another study reported that an NIHSS score of $\geq 8$ and left atrial diameter of $\geq 3.8 \mathrm{~cm}$ on transthoracic echocardiography in patients with acute-phase cerebral infarction are independent relevant factors for covert atrial fibrillation. ${ }^{8)}$ In the present case, the left atrial diameter was $3.9 \mathrm{~cm}$, and anticoagulant therapy was selected under a tentative diagnosis of covert atrial fibrillation. However, according to a previous study, ${ }^{6}$ the detection rate of covert atrial fibrillation on electrocardiography at the time of admission is $2.7 \%$, and the cumulative detection rate is $17.5 \%$ even when 24-hour Holter's electrocardiography/frequent electrocardiography for 5 days/subsequent extracorporeal electrocardiographic monitoring for 7 days are performed. Recently, covert atrial fibrillation has been detected using an insertable cardiac monitoring device, but a previous study reported the detection rates at 6,12 , and 36 months to be $8.9,12.4$, and $30.0 \%$, respectively. ${ }^{7)}$ The possibility that covert atrial fibrillation can be diagnosed during a short admission period is low, and anticoagulant therapy is continued to prevent recurrence until the detection of atrial fibrillation in many cases.

The morbidity rate of HHT is $1 / 5000$ to 8000 persons, ${ }^{9}{ }^{9}$ and this disease develops through autosomal dominant inheritance, with a permeability of $97 \%$. Known causative gene mutations are $9 q$ chromosome (9q33-q34) endoglin (ENG) and $12 \mathrm{q}$ chromosome $(12 \mathrm{q} 11-\mathrm{q} 14)$ active receptorlike kinase type 1 (ALK1). These are termed HHT1 and HHT2, respectively. Furthermore, HHT with juvenile polyposis was reported, and 18q chromosome (18q21) Small Mothers Against Decapentaplegic Homolog 4 (SMAD4) gene mutations were confirmed. ${ }^{9,10)}$ The disease type is evaluated as type 1 or 2 HHT in $85-90 \%$ of patients. In Japan, type 1 HHT is frequently observed, and it is likely to be revealed by cerebral or pulmonary lesions at a young age. ${ }^{9)}$ Patients meeting $\geq 3$ of Curacao's diagnostic criteria: (1) repeated nasal hemorrhage, (2) capillary (malformation) of the lips/oral cavity/fingers/nose, (3) capillary (malformation) of the digestive tract or arteriovenous malformation of the lungs/liver/brain/spinal cord, and (4) first-degree relatives with HHT, which were proposed by Shovlin et al., are definitively diagnosed with HHT, and those meeting $\geq 2$ of these criteria are tentatively diagnosed with HHT. ${ }^{11}$ ) Clinically, our patient met three of these criteria, leading to a definitive diagnosis of HHT. In a previous study, ${ }^{12)}$ cerebral lesions and lung lesions were shown in $11 \%$ and $33 \%$ of HHT, respectively. HHT of the lungs is complicated by pulmonary arteriovenous fistulae in many cases, with an incidence of $15-50 \% .{ }^{13)}$ A pulmonary arteriovenous fistula is defined as an abnormal shunt between the pulmonary artery and vein. As clinical symptoms, a right-to-left shunt causes chronic hypoxemia, leading to cyanosis or erythrocytosis. As results of a right-to-left shunt, brain abscess and paradoxical cerebral embolism are observed. ${ }^{14)}$ However, in the present case, these symptoms were absent, and there was no excessive hypoxemia; therefore, the presence of a pulmonary arteriovenous fistula was not suspected based on physical signs or hematological data in the initial phase of treatment.

According to a previous study in Japan, cerebral embolism related to pulmonary arteriovenous fistulae accounts 
for $0.5 \%$ of patients with cerebral infarction. ${ }^{15)}$ Another study reported that the source of embolism was a deep venous thrombus or an intra-fistula thrombus related to blood stagnation. ${ }^{16)}$ When detecting pulmonary arteriovenous fistulae using contrast echocardiography, false-positive findings are included. ${ }^{17)}$ One previous study suggested that even minor pulmonary arteriovenous fistulae induce intra-fistula thrombus formation. ${ }^{18)}$ Therefore, in the present case, additional contrast echocardiography was not performed after the identification of pulmonary arteriovenous fistulae on thoracic CT. Currently, endovascular treatment is selected as the first-choice procedure for pulmonary arteriovenous fistulae. In the International Guidelines for the Diagnosis and Management of Hereditary Hemorrhagic Telangiectasia in 2009 , it is described that treatment may also be indicated for pulmonary arteriovenous fistulae with a feeding artery diameter of $\geq 3 \mathrm{~mm} .{ }^{13)}$ Furthermore, Haga et al. performed catheter embolization to prevent recurrent cerebral infarction in patients with pulmonary arteriovenous fistula with a feeding artery diameter of $\leq 2 \mathrm{~mm}$, and reported a favorable course. ${ }^{18)}$ In the present case, fine pulmonary arteriovenous fistulae were also observed at the time of treatment, and catheter embolization of all fistulae was conducted considering the risk of embolism related to intra-fistula thrombus formation. Moreover, HHT has the characteristics of hemorrhagic disease. Repeated nasal hemorrhage comprises the greater portion of HHT-associated hemorrhage. ${ }^{14)}$ Our patient had a history of nasal hemorrhage during childhood, but its frequency/degree was low/mild; therefore, HHT were not suspected. In addition, there was no nasal hemorrhage from the admission period involving anticoagulant therapy until treatment for pulmonary arteriovenous fistulae. Previous studies reported gastrointestinal hemorrhage as a hemorrhagic event other than nasal hemorrhage. Suzuki et al. found that the most frequent site of hemorrhage was the stomach (93.7\%), although capillary telangiectasia of the digestive tract varied. ${ }^{19)}$ In the present case, nasal hemorrhage was not confirmed before or after admission, and capillary telangiectasia of the stomach and ascending colon was observed. In addition, a fecal blood test was positive that was detected at the onset of severe weakness, and gastrointestinal hemorrhage was considered to be an etiological factor for anemia. A previous study of antithrombotic therapy involving 379 patients with HHT demonstrated no exacerbation of nasal hemorrhage in $40.4 \%$. Hemorrhage other than nasal hemorrhage was noted in $13.1 \%$. In the anticoagulant therapy group, the percentage was $19.5 \%$, being double or greater than that in the antiplatelet therapy group $(8.8 \%)$. In the present case, there was no persistent hemorrhage on endoscopy, and hemostasis for capillary telangiectasia of the gastrointestinal mucosa was not performed. However, there was no progression of anemia during a 1-year period of follow-up after the completion of anticoagulant therapy; therefore, anticoagulant therapy may have been involved in the progression of anemia in the presence of HHT. ${ }^{20)}$ In HHT, patients with pulmonary arteriovenous fistulae who develop cerebral embolism, it is necessary to promptly treat pulmonary arteriovenous fistulae for the early completion of anticoagulant therapy considering the risk of hemorrhage. To make a diagnosis of HHT in the early phase, it may be necessary to accurately inquire about the history of nasal hemorrhage or family history, which is the most basic method. In addition, chest X-ray films must be thoroughly evaluated when reviewing the etiology of ESUS, as pulmonary arteriovenous fistulae can be detected by retrospectively confirming chest X-ray films.

\section{Conclusion}

We reported a patient in whom anticoagulant therapy after thrombectomy induced anemia, leading to a diagnosis of HHT. With the widespread application of thrombectomy, the incidence of cardiogenic embolism has increased, and anticoagulant therapy is selected to prevent recurrence in many cases. However, it may be important to inquire from the patients, which is the simplest and most useful method for HHT diagnosis, and thoroughly evaluate chest X-ray films considering the presence of rare diseases, such as HHT, as a background factor for cerebral embolism.

\section{Disclosure Statement}

We declare no conflict of interest.

\section{References}

1) Yoshimura S, Sakai N, Okada Y, et al: Efficacy of endovascular treatment for acute cerebral large-vessel occlusion: analysis of nationwide prospective registry. J Stroke Cerebrovasc Dis 2014; 23: 1183-1190.

2) Hart RG, Diener HC, Coutts SB, et al: Embolic strokes of undetermined source: the case for a new clinical construct. Lancet Neurol 2014; 13: 429-438.

3) Kitagawa K: Pathophysiology of ESUS (embolic stroke of undetermined sources). Neurol Therap 2016; 33: 382-386.

4) Toyoda K, Okumura K, Hashimoto Y, et al: Cryptogenic ischemic stroke and embolic stroke of undetermined 
source: clinical implications and importance for detection of covert atrial fibrillation in Japan. Jpn J Stroke 2016; 38: 77-85. (in Japanese)

5) Gladstone DJ, Spring M, Dorian P, et al: Atrial fibrillation in patients with cryptogenic stroke. $N$ Engl J Med 2014; 370: 2467-2477.

6) Jabaudon D, Sztajzel J, Sievert K, et al: Usefulness of ambulatory 7-day ECG monitoring for the detection of atrial fibrillation and flutter after acute stroke and transient ischemic attack. Stroke 2004; 35: 1647-1651.

7) Sanna T, Diener HC, Passman RS, et al: Cryptogenic stroke and underlying atrial fibrillation. N Engl J Med 2014; 370: 2478-2486.

8) Fujii S, Shibazaki K, Iguchi Y, et al: [Relationship between left atrial size and paroxysmal atrial fibrillation in acute ischemic stroke.] Clin Neurol 2009; 49: 629-633. (in Japanese)

9) Dakeishi M, Shioya T, Wada Y, et al: Genetic epidemiology of hereditary hemorrhagic telangiectasia in a local community in the northern part of Japan. Hum Mutat 2002; 19: $140-148$

10) Gallione CJ, Repetto GM, Legius E, et al: A combined syndrome of juvenile polyposis and hereditary haemorrhagic telangiectasia associated with mutations in MADH4 (SMAD4). Lancet 2004; 363: 852-859.

11) Shovlin CL, Guttmacher AE, Buscarini E, et al: Diagnostic criteria for hereditary hemorrhagic telangiectasia (RenduOsler-Weber syndrome). Am J Med Genet 2000; 91: 66-67.

12) Haitjema $T$, Disch F, Overtoom TT, et al: Screening family members of patients with hereditary hemorrhagic telangiectasia. Am J Med 1995; 99: 519-524.
13) Faughman ME, Palda VA, Garsia-Tsao, et al: International guidelines for the diagnosis and management of hereditary haemorrhagic telangiectasia. J Med Genet 2011; 48: $73-87$.

14) Swanson KL, Prakash UB, Stanson AW: Pulmonary arteriovenous fistulas: Mayo Clinic experience, 1982-1997. Mayo Clin Proc 1999; 74: 671-680.

15) Kimura K, Minematsu K, Nakajima M: Isolated pulmonary arteriovenous fistula without Rendu-Osler-Weber disease as a cause of cryptogenic stroke. J Neurol Neurosurg Psychiatry 2004; 75: 311-313.

16) Devuyst G, Boggusslavasky J: Pulmonary arteriovenous fistula. In: Uncommon causes of stroke, First ed, Bogousslavasky J, Caplan L. Cambridge University Press 2001: 198-205.

17) Kuriki A, Katou H, Mizuma K, et al: Clinical features of ischemic stroke and transient ischemic attack associated with suspicious pulmonary arteriovenous fistula detected only by transesophageal echocardiography. Neurosonology 2015: 28: 134-138.

18) Haga $T$, Takahira $K$, Ono H, et al: A case of small pulmonary arteriovenous malformation diagnosed after juvenile stroke. Shinzo 2013; 45: 1421-1427. (in Japanese)

19) Suzuki M, Kobayashi O, Ohta K, et al: A case report of hereditary hemorrhagic telangiectasia (HHT) with reccurent gastric bleeding. Prog Dig Endosc 2015; 86: $138-139$.

20) Devlin HL, Hosman AE, Shovlin CL: Antiplatelet and anticoagulant agents in hereditary hemorrhagic telangiectasia. N Engl J Med 2013; 368: 876-878. 